\title{
Organic fragments from graphene oxide: Isolation, characterization and solvent effects
}

\author{
RAVULA THIRUPATHI ${ }^{\mathrm{a}, \mathrm{b}, \mathrm{c}}$, Y JAYASUBBA REDDY ${ }^{\mathrm{b}}$, ERODE N PRABHAKARAN $^{\mathrm{d}, *}$ and \\ HANUDATTA S ATREYA*,b \\ ${ }^{a}$ Institute Nanoscience Initiative, Indian Institute of Science, Bangalore 560 012, India \\ ${ }^{\mathrm{b}}$ NMR Research Centre, Indian Institute of Science, Bangalore 560 012, India \\ ${ }^{\mathrm{c}}$ Solid State and Structural Chemistry Unit, Indian Institute of Science, Bangalore 560 012, India \\ ${ }^{\mathrm{d}}$ Department of Organic Chemistry, Indian Institute of Science, Bangalore 560 012, India \\ e-mail: erodeprabhakaran@gmail.com; hsatreya@sif.iisc.ernet.in
}

MS received 18 November 2013; revised 6 December 2013; accepted 7 December 2013

\begin{abstract}
As-prepared graphene oxide (GO) contains oxidative debris which can be washed using basic solutions. We present the isolation and characterization of these debris. Dynamic light scattering (DLS) is used to monitor the separation of the debris in various solvents in the presence of different protic and aprotic alkylamino bases. The study reveals that the debris are rich in carbonyl functional groups and water is an essential component for separation and removal of the debris from GO under oxidative reaction conditions.
\end{abstract}

Keywords. Graphene oxide; oxidative debris; solid state NMR; dynamic light scattering.

\section{Introduction}

Graphene oxide (GO) has attracted significant recent interest in diverse applications. ${ }^{1-4}$ It also serves as a potential intermediate for the large-scale preparation of graphene. ${ }^{5}$ The wide applicability for GO comes from its superior solubility in a variety of solvents, especially in water. ${ }^{6}$ In recent years, several groups have reported the observation of oxidative debris in GO under different conditions. ${ }^{7-9}$ The model proposed for GO-debris interaction ${ }^{7}$ considers the debris to act similar to a surfactant rendering the dispersion of GO stable in water. Interaction between debris and GO has been proposed to be non-covalent in nature with contributions from hydrogen bonding and $\pi$-stacking interactions. ${ }^{7}$ Existence of such debris on GO sheets has been shown to alter its nature and reactivity. ${ }^{8}$ However, despite being extensively investigated ${ }^{10}$ there remains ambiguity on the nature of the debris. ${ }^{6,11}$ Further, separation of debris requires subjecting $\mathrm{GO}$ to oxidative reaction conditions in the presence of different bases. ${ }^{7,9}$ Dependence of the nature of debris on the conditions of the oxidative reactions and hence the process of separation, remain unclear. We make use of dynamic light scattering (DLS) to monitor the separation of debris from GO in various solvents in the presence of different bases. Separation of debris and hence its isolation occurs only under

*For correspondence aqueous conditions. We have characterized the debris separated from GO using infra-red and solid state NMR spectroscopy. Overall, the study reveals that the debris are rich in carbonyl functional groups and water is an essential component for their separation and removal from GO under oxidative reaction conditions.

\section{Experimental}

In order to isolate and characterize the debris, a goldcoloured suspension of GO $(1 \mathrm{mg} / \mathrm{ml})$ (prepared by the Hummer's method ${ }^{12}$ ) (figure 1a), was refluxed at boiling point of water in a mild base, aqueous ammonia (aq. $\mathrm{NH}_{3}$ ), for $1 \mathrm{~h}$. The resulting black suspension was allowed to settle for $12 \mathrm{~h}$ and DLS ${ }^{13,14}$ data was obtained for the resulting supernatant. DLS data showed the presence of two components (figure 1b) whose relative spatial dimensions differed by more than two orders of magnitude. The smaller component $(5 \pm$ $2 \mathrm{~nm}$ ) was assigned to the debris. The larger sized particles were termed as base-treated GO (BGO). BGO are of comparable size, but slightly smaller than GO (figure 1b). Similar results were obtained when GO was treated with other aqueous basic solutions (figure 1c, entries 1-4). For a detailed structural characterization, we isolated debris formed from aq- $\mathrm{NH}_{3}$-treated $\mathrm{GO}$ (figure $1 \mathrm{~b}$ ) by passing the mixture through a $0.2 \mu \mathrm{m}$ filter. Structures of the fragments and BGO thus obtained (BGO-A; letter A corresponds to the specific base used: 


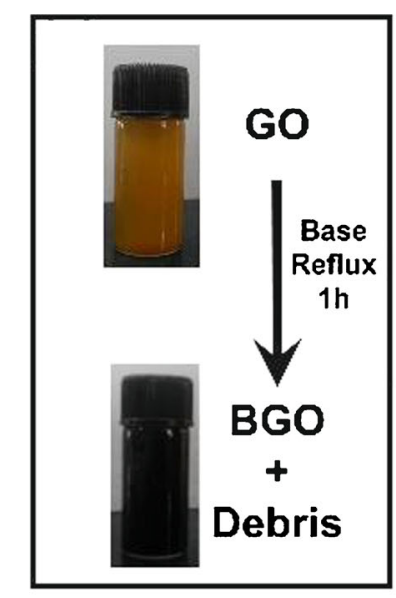

(a)

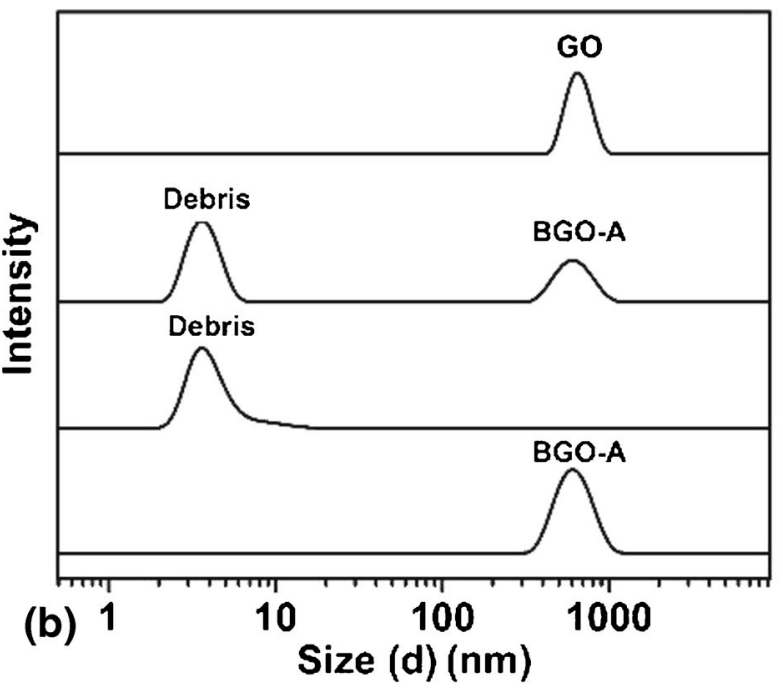

\begin{tabular}{cccc}
\hline Sl.No & Reaction $\left(\right.$ code $\left.^{\mathrm{a}}\right)$ & Base in solution & Debris $^{\mathrm{b}}$ \\
\hline 1 & $\mathrm{NH}_{3}+\mathrm{H}_{2} \mathrm{O}(\mathrm{BGO}-\mathrm{A})$ & $\mathrm{NH}_{3}, \mathrm{OH}^{-}$ & $\checkmark$ \\
2 & $\mathrm{NaOH}+\mathrm{H}_{2} \mathrm{O}$ & $\mathrm{OH}^{-}$ & $\checkmark$ \\
3 & $\mathrm{DBU}+\mathrm{H}_{2} \mathrm{O}$ & $\mathrm{DBU}, \mathrm{OH}^{-}$ & $\checkmark$ \\
4 & $50 \% \mathrm{MeOH} / \mathrm{H}_{2} \mathrm{O}+\mathrm{DBU}$ & $\mathrm{DBU}, \mathrm{OH}^{-}$ & $\checkmark$ \\
6 & $\mathrm{DBU}+\mathrm{MeOH}$ & $\mathrm{DBU}$ & $\times$ \\
7 & DIEA + NMP $(\mathrm{BGO}-\mathrm{D}$ NMP $)$ & $\mathrm{DIEA}$ & $\times$ \\
8 & Ethylenediamine(EDA $)+\mathrm{MeOH}$ & $\mathrm{EDA}$ & $\times$ \\
\hline
\end{tabular}

(c)

\section{${ }^{\mathrm{a}}$ Represents leftover GO \\ ${ }^{\mathrm{b}}$ Based on DLS experiment}

Figure 1. (a) Photographic image of the gold-coloured suspension of GO in water before and after refluxing with base (aq. $\mathrm{NH}_{3}$ ). (b) Size distributions of particles as observed in DLS: GO, BGO-A + debris, debris and BGO-A. (c) Table showing the presence/absence of types of bases in different solutions used in the current study and the separation of debris exclusively when water was present as solvent.

ammonia as shown in figure 1c) were characterized using FTIR, NMR and TGA.

The FTIR emission spectrum of GO (figure 2) shows the characteristic broad band $\left(3000-3600 \mathrm{~cm}^{-1}\right)$ centered at around $3500 \mathrm{~cm}^{-1}$ (corresponding to a combination of the stretching bands of $-\mathrm{O}-\mathrm{H}$ in $-\mathrm{COOH}$, $-\mathrm{C}(\mathrm{O}-\mathrm{H})=$ and $\mathrm{H}-\mathrm{O}-\mathrm{H})$ and sharp characteristic peaks at $1720 \mathrm{~cm}^{-1}, 1618 \mathrm{~cm}^{-1}, 1399 \mathrm{~cm}^{-1}$ and $1225 \mathrm{~cm}^{-1}$ corresponding to the asymmetric stretching of carboxylic and carbonyl $\mathrm{C}=\mathrm{O}$, alcoholic $\mathrm{C}-\mathrm{O}$ and epoxy $\mathrm{C}-\mathrm{O}$ groups, respectively. ${ }^{15}$ These functional groups are known to be present on the GO surface, clustered together in the $\mathrm{sp}^{3}$ clusters. ${ }^{16}$ In BGO-A (figure 2), the carboxylic $\mathrm{C}=\mathrm{O}$ band at $\sim 1720 \mathrm{~cm}^{-1}$ is absent. This is expected since the peripheral carboxylic acids are converted to amides upon refluxing with aq. $\mathrm{NH}_{3}$. 


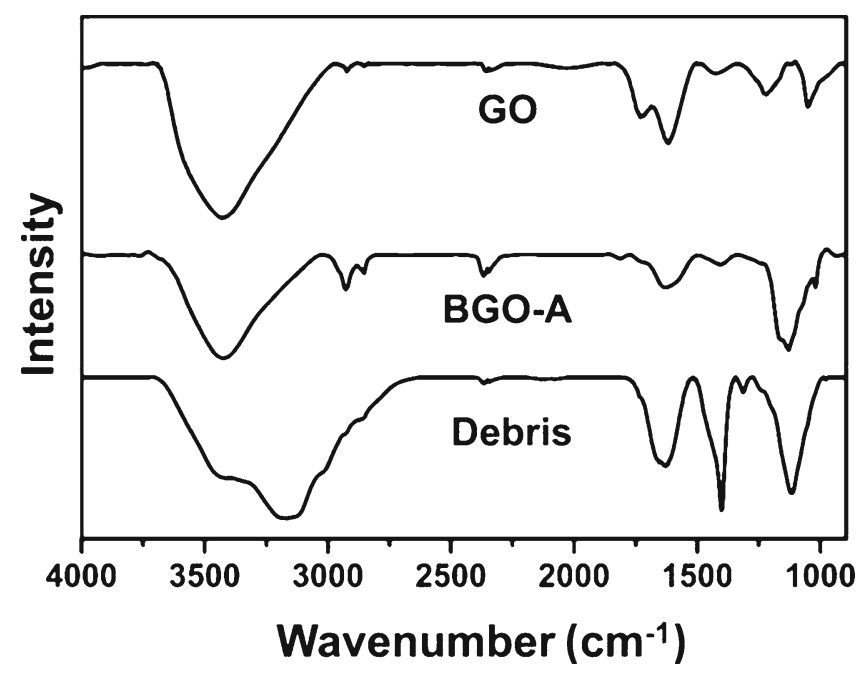

Figure 2. FTIR spectra of GO, BGO-A and debris.

The debris sample (figure 2) showed an additional redshifted band centered at $\sim 3200 \mathrm{~cm}^{-1}$, in the region of primary amide N-H stretch. Appearance of strong bands corresponding to primary amide carbonyl stretch at $1650 \mathrm{~cm}^{-1}$, amide N-H bend at $1400 \mathrm{~cm}^{-1}$ and amide $\mathrm{C}-\mathrm{N}^{17}$ stretch at $1100 \mathrm{~cm}^{-1}$ are further supportive of the presence of carbonyls and primary amide groups in the debris.

Thermo gravimetric analysis (TGA) of GO showed $36 \%$ weight loss in the region of $150^{\circ} \mathrm{C}$ to $300^{\circ} \mathrm{C}$ due to the decomposition of labile oxygen containing functional groups. ${ }^{16,18}$ Notably, BGO-A shows higher thermal stability ${ }^{19}$ than GO, presumably due to the loss of the reactive/unstable functional groups such as the epoxy and carboxylic acid groups upon treatment of GO with $\mathrm{NH}_{3}$. The TGA of debris (see supplementary information figure S7) revealed complete weight loss of the material after $600^{\circ} \mathrm{C}$, unlike GO and BGO-A samples (in which complete weight loss was not observed up to $750^{\circ} \mathrm{C}$ ), suggesting that there are no graphitic regions left on the debris.

While the FTIR data gives an overall indication of the presence/absence of the different functional groups, relative abundance of $\mathrm{C}=\mathrm{O}$ (amide) and $\mathrm{C}=\mathrm{C}$ (aromatic) groups in debris or BGO-A remains ambiguous due to spectral overlap and/or weak absorptivity, especially of $\mathrm{C}=\mathrm{C}$ (aromatics). Such insights can be obtained from NMR spectroscopy which has distinct chemical shifts for $\mathrm{C}=\mathrm{O}$ and $\mathrm{C}=\mathrm{C}$ functional groups. Figure 3 shows two sets of three ${ }^{13} \mathrm{C}$ magic angle spinning (MAS) NMR spectra for GO, BGO-A and debris acquired in the solid state. While the ${ }^{13} \mathrm{C}$ MAS spectra (figure 3a) involve direct excitation and detection of all ${ }^{13} \mathrm{C}$ nuclei, the ${ }^{13} \mathrm{C} \mathrm{CP}-\mathrm{MAS}$ spectra (figure $3 b$ ) involve ${ }^{1} \mathrm{H}_{-}{ }^{13} \mathrm{C}$ polarization transfer ${ }^{16,20,21}$ and provide selective information on ${ }^{13} \mathrm{C}$ nuclei that are proximal or directly attached to hydrogen atoms. In figure $3 \mathrm{a}$, peaks centered at $\sim 60$ and $\sim 70 \mathrm{ppm}$ in ${ }^{13} \mathrm{C}$ MAS spectrum of GO are assigned to carbons containing epoxy and hydroxyl groups, respectively. ${ }^{16,22}$ The broad peak observed at 120-140 ppm corresponds to the aromatic $\mathrm{sp}^{2}$ carbon, and the low intensity peaks at $\sim 160$ and $\sim 200 \mathrm{ppm}$ are attributed to the carboxyl and amide carbonyl carbons, respectively, which lie along the periphery of GO sheets. ${ }^{21,22}$ The ${ }^{13} \mathrm{C}$ NMR spectrum of BGO-A (figure $3 \mathrm{~b}$ ) exhibits a notable decrease in relative intensity of the $60-70 \mathrm{ppm}$ peaks (relative to the aromatic carbons) suggesting significant loss of C$\mathrm{O}$ groups (both $\mathrm{C}-\mathrm{OH}$ and $\mathrm{C}-\mathrm{O}-\mathrm{C}$, indicated by arrow in figure 3a). Further, there is appearance of a new, relatively higher intensity peak at $\sim 170$ ppm in BGOA, indicating an increase in the amide carbonyl groups upon treatment with the base. These observations corroborate the FTIR data, suggesting the formation of primary amides in BGO-A carboxylic acid (peripheral) groups of GO.

\section{Discussion}

The ${ }^{13} \mathrm{C}$-MAS spectrum of the debris (figure 3a) exhibits a strong peak at $\sim 163$ ppm and relatively lowintensity peaks in the range of $165-170 \mathrm{ppm}$. There is a significant decrease in the relative intensity of $13 \mathrm{C}$ peaks in aliphatic C-O region (i.e., 60-70 ppm) indicating that the epoxy and alcohol groups are present in very small amounts. Further, the absence of peaks at $120-140 \mathrm{ppm}$ also indicates the absence of aromatic $\left(\mathrm{sp}^{2}\right)$ carbons in the debris. Enhanced intensity of the peaks at $165-170 \mathrm{ppm}$ in the ${ }^{13} \mathrm{C}$ CP-MAS (figure $3 \mathrm{~b}$ ) indicates the presence of amide groups, peaks corresponding to which are also seen in ${ }^{13} \mathrm{C}$-MAS spectrum of debris (figure 3a). Notably, the sharp peak at $\sim 163$ ppm observed in ${ }^{13} \mathrm{C}$-MAS is absent in the CPMAS spectrum (see figure S8 of supplementary information showing a comparison of ${ }^{13} \mathrm{C}-\mathrm{MAS}$ and $\mathrm{CP}$ MAS spectrum) suggesting the additional presence of a carbonyl moiety that is devoid of proximal protons, namely the $\mathrm{CO}_{3}^{2-}$ group in the debris. Our assignment of this peak ( $\sim 163 \mathrm{ppm})$ to $\mathrm{CO}_{3}^{2-}$ is supported ${ }^{23}$ further by the ${ }^{13} \mathrm{C}$ MAS spectral data of $\left(\mathrm{NH}_{4}\right)_{2} \mathrm{CO}_{3}$ which exhibited a peak at $\sim 163 \mathrm{ppm}$ (see Supporting Information figure S1). Formation of $\mathrm{CO}_{3}^{2-}$ upon treatment of $\mathrm{GO}$ with base has recently been proposed to occur through the decarboxylation reaction. ${ }^{11}$ Remarkably, treatment of GO with an organic non-nucleophilic base N,N diisopropylethylamine (DIEA) in an anhydrous organic solvent N-methyl-2-pyrrolidone (NMP) did not result in any by-products like debris. This suggests that the 

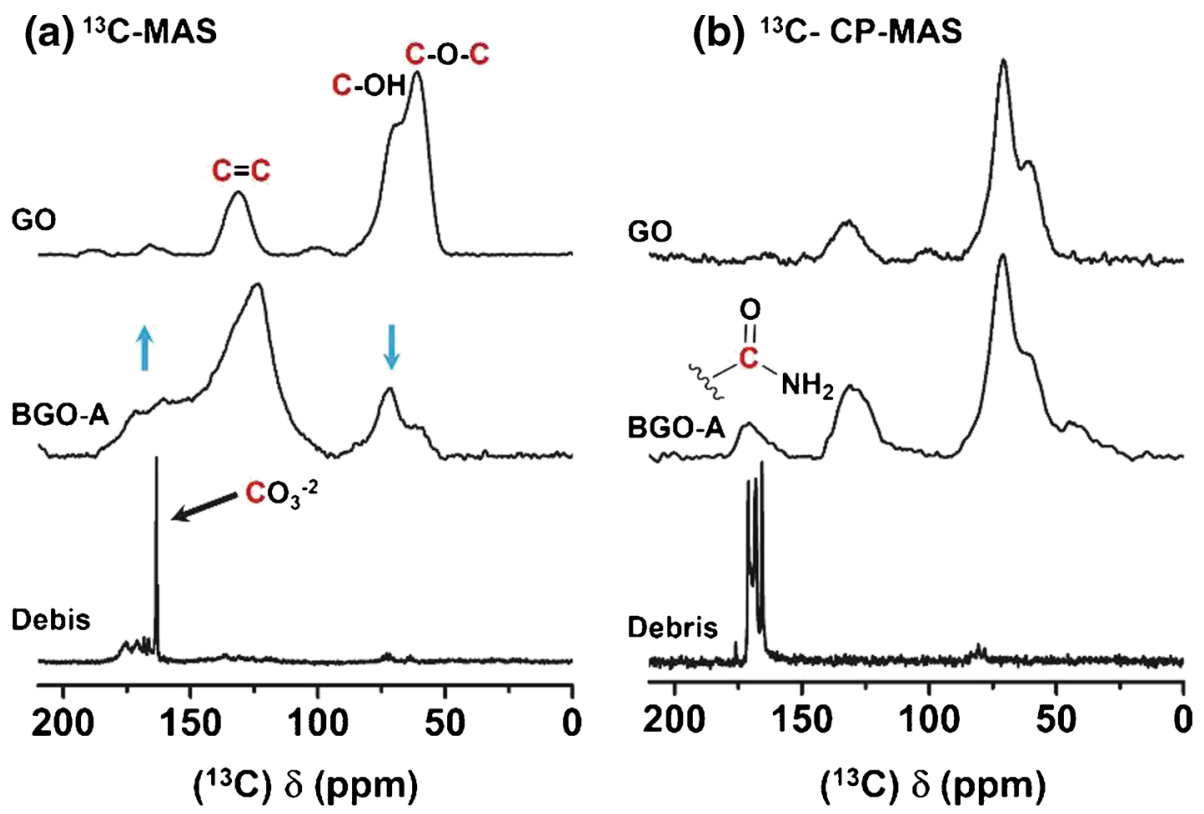

Figure 3. Solid state ${ }^{13} \mathrm{C}-\mathrm{MAS}$ NMR spectra of (a) GO, BGO-A and debris. ${ }^{13} \mathrm{C} \mathrm{CP}-$ MAS spectrum of (b) GO, BGO-A and debris acquired at a ${ }^{1} \mathrm{H}$ resonance frequency of $500 \mathrm{MHz}$.

separation of the debris is nucleophile-driven reaction as explained in the following sections.

Separation of carbonyl-rich small organic fragments (debris) can be explained by considering the chemical environment prevailing in a suspension of GO in aqueous ammonia. Aqueous ammonia contains three species: $\mathrm{NH}_{3}$ and $\mathrm{NH}_{4}^{+}$resulting from association of $\mathrm{NH}_{3}$ with $\mathrm{H}^{+}$in the amphoteric water and $\mathrm{OH}^{-}$whose concentration increases with the concentration of ammonia in the medium. Concentration of the $\mathrm{NH}_{4}^{+}$ions will be significantly low under these conditions. Both $\mathrm{NH}_{3}$ and $\mathrm{OH}^{-}$, present in relatively high amounts, could serve as Bronsted base and can generate negative charge on debris as well as on GO thus separating debris from GO. ${ }^{7}$ DLS data showed that the size of debris obtained under these conditions (aq. $\mathrm{NH}_{3}$ ) were comparable to those obtained from other aqueous basic solutions, where ammonia is replaced with more basic and hindered nucleophilic organic bases such as N,N-diisopropylethylamine (DIPEA), diazabicycloundecene (DBU), 4-dimethylaminopyridine (DMAP), etc. (figure 1c, figure S3 in supplementary information.). On the other hand, the debris from aq. $\mathrm{NH}_{3}$ are larger than those obtained from aqueous $\mathrm{NaOH}$, where $\mathrm{OH}^{-}$ions are the only base and are found in greater abundance than in aq. $\mathrm{NH}_{3}$ solution. These results clearly indicate that the hydroxyl ion is responsible for the separation of debris.

Interestingly, treatment of GO with the tertiary amines DIEA and DBU in the anhydrous organic solvent NMP and $\mathrm{MeOH}$, respectively, do not result any observable fractions of debris under identical conditions (figure 1c). Additionally, FTIR characterization

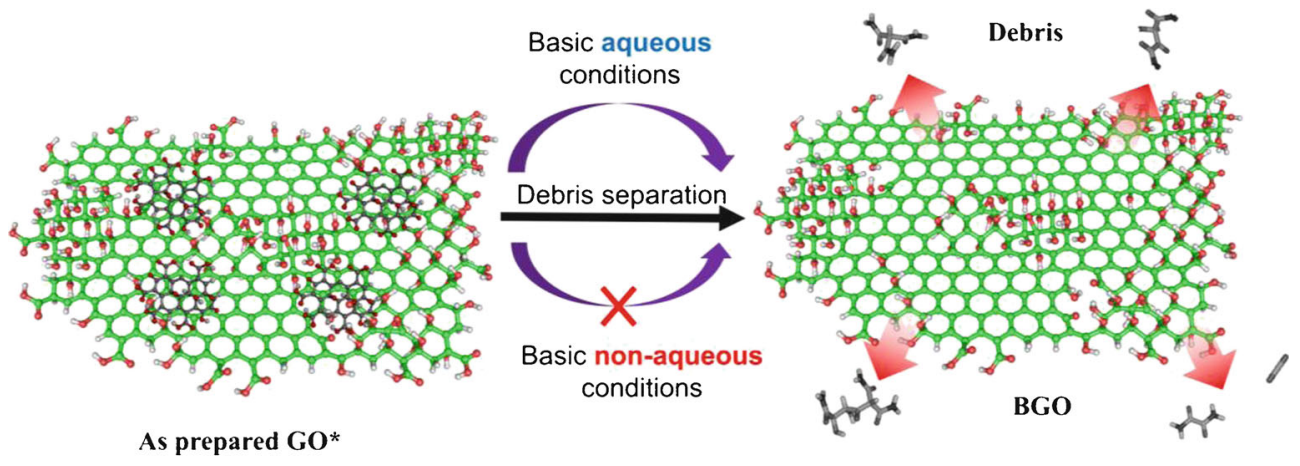

Figure 4. Schematic ball and stick representation depicting the separation of debris from GO exclusively under aqueous basic conditions (green ball-carbon in GO basal plane; grey ball—carbon in debris; blue ball—nitrogen; red ball—oxygen; white ball—hydrogen. * The as-prepared GO is representative of the model proposed by Rourke et al. ${ }^{7}$ ). 
of BGO obtained from treatment with DIEA (BGO$\mathrm{D}_{N M P}$ ) in NMP (a non-nucleophilic neutral organic solvent) showed the presence of all the native functional groups of $\mathrm{GO}(\mathrm{C}-\mathrm{O}-\mathrm{C}, \mathrm{C}-\mathrm{OH}, \mathrm{C}=\mathrm{O})$ in similar fractions as that of GO (see supplementary information figure S4). XPS also showed similar preservation of all the $\mathrm{C}-\mathrm{O}$ containing species in $\mathrm{BGO}-\mathrm{D}_{N M P}$ (see supplementary information figure S5). Both observations point to the fact that there are no perceivable changes in functional group content upon refluxing of GO with DIPEA in NMP.

\section{Conclusion}

In conclusion, current study provides an understanding of a method for separation of debris from GO sheets and the crucial functional group characterization of these debris. Washing process for the removal of debris from $\mathrm{GO}$ in the presence of a base requires water as the solvent, since similar debris separation does not occur under non-aqueous conditions (figure 4). The separated debris are rich in amide functional groups. However, non-occurrence of this separation process in the presence of bases in anhydrous solvents under identical temperatures and conditions suggests that separation of debris from GO is accomplished through chemical reactions. Separation of debris has its origins in the nucleophilic substitution of functional groups on the GO surface with aqueous bases. This study sheds new light on the fundamental understanding of debris-GO interaction, enabling better control over functionalization of the GO surface.

\section{Supplementary information}

Experimental details, Characterization techniques, DLS, XPS are given as supplementary information (figures S1-S9). For details see www.ias.ac.in/chemsci.

\section{Acknowledgements}

The Nanoscience Centre at Indian Institute of Science (IISc) is gratefully acknowledged for characterization facilities. RT and JYR thank the Council of Scientific and Industrial Research (CSIR) for fellowship.

\section{References}

1. Wan X, Huang Y and Chen Y 2012 Acc. Chem. Res. 45 598

2. Balapanuru J, Yang J-X, Xiao S, Bao Q, Jahan M, Polavarapu L, Wei J, Xu Q-H and Loh K P 2010 Angew. Chem. Int. Ed. 496549

3. Jung J H, Cheon D S, Liu F, Lee K B and Seo T S 2010 Angew. Chem. Int. Ed. 495708

4. Liu Z, Robinson J T, Sun X and Dai H $2008 \mathrm{~J}$. Am. Chem. Soc. 13010876

5. Park S and Ruoff R S 2009 Nat. Nanotechnol. 4217

6. Dreyer D R, Park S, Bielawski C W and Ruoff R S 2010 Chem. Soc. Rev. 39228

7. Rourke J P, Pandey P A, Moore J J, Bates M, Kinloch I A, Young R J and Wilson N R 2011 Angew. Chem. Int. Ed. 50, 3173

8. Su C, Acik M, Takai K, Lu J, Hao S-J, Zheng Y, Wu P, Bao Q, Enoki T, Chabal Y J and Ping Loh K 2012 Nat. Commun. 31298

9. He W and Lu L 2012 Adv. Funct. Mater. 22

10. Erickson K, Erni R, Lee Z, Alem N, Gannett W and Zettl A 2010 Adv. Mater. 224467

11. Dimiev A M, Alemany L B and Tour J M 2012 ACS Nano. 7576

12. Hummers W S and Offeman R E $1958 \mathrm{~J}$. Am. Chem. Soc. 801339

13. Stankovich S, Piner R D, Nguyen S T and Ruoff R S 2006 Carbon 443342

14. DLS assumes the particles to be of spherical shape and hence the dimensions mentioned above cannot be taken as direct lateral dimensions of the sheets

15. Szabó T, Berkesi O, Forgó P, Josepovits K, Sanakis Y, Petridis D and Dékány I 2006 Chem. Mater. 182740

16. Lerf A, He H, Forster M and Klinowski J 1998 J. Phys. Chem. B $\mathbf{1 0 2} 4477$

17. Hu C, Liu Y, Yang Y, Cui J, Huang Z, Wang Y, Yang L, Wang H, Xiao Y and Rong J 2013 J. Mater. Chem. B 139

18. Stankovich S, Dikin D A, Piner R D, Kohlhaas K A, Kleinhammes A, Jia Y, Wu Y, Nguyen S T and Ruoff R S 2007 Carbon 451558

19. The thermal stability of the samples were directly correlated with their first transition temperatures. Please see supplementary Information figure S7.

20. He H, Klinowski J, Forster M and Lerf A 1998 Chem. Phys. Lett. 28753

21. Casabianca L B, Shaibat M A, Cai W W, Park S, Piner R, Ruoff R S and Ishii Y 2010 J. Am. Chem. Soc. 132 5672

22. Cai W, Piner R D, Stadermann F J, Park S, Shaibat M A, Ishii Y, Yang D, Velamakanni A, An S J, Stoller M, An J, Chen D and Ruoff R S 2008 Science 3211815

23. Papenguth H W, Kirkpatrick R J and Sandberg P A 1989 Am. Min. 741152 